\title{
The Neuroeconomics of Alcohol Demand: An Initial Investigation of the Neural Correlates of Alcohol Cost-Benefit Decision Making in Heavy Drinking Men
}

\author{
James MacKillop*,',2, Michael T Amlung',3, John Acker', Joshua C Gray', Courtney L Brown', \\ James G Murphy ${ }^{2,4}$, Lara A Ray ${ }^{5}$ and Lawrence H Sweet ${ }^{1,6}$ \\ 'Department of Psychology, University of Georgia, Athens, GA, USA; ²Department of Behavioral and Social Sciences, Brown University, Providence, \\ RI, USA; ${ }^{3}$ Department of Psychological Sciences, University of Missouri, Columbia, MO, USA; ${ }^{4}$ Department of Psychology, University of Memphis, \\ Memphis, TN, USA; ${ }^{5}$ Department of Psychology, University of California, Los Angeles, Los Angeles, CA, USA; ${ }^{6}$ Department of Psychiatry and \\ Human Behavior, Brown University, Providence, RI, USA
}

Neuroeconomics integrates concepts and methods from psychology, economics, and cognitive neuroscience to understand how the brain makes decisions. In economics, demand refers to the relationship between a commodity's consumption and its cost, and, in behavioral studies, high alcohol demand has been consistently associated with greater alcohol misuse. Relatively little is known about how the brain processes demand decision making, and the current study is an initial investigation of the neural correlates of alcohol demand among heavy drinkers. Using an event-related functional magnetic resonance imaging (fMRI) paradigm, participants ( $N=24)$ selected how much they would drink under varying levels of price. These choices determined access to alcohol during a subsequent bar laboratory self-administration period. During decisions to drink in general, greater activity was present in multiple distinct subunits of the prefrontal and parietal cortices. In contrast, during decisions to drink that were demonstrably affected by the cost of alcohol, significantly greater activation was evident in frontostriatal regions, suggesting an active interplay between cognitive deliberation and subjective reward value. These choices were also characterized by significant deactivation in default mode network regions, suggesting suppression resulting from greater cognitive load. Across choice types, the anterior insula was notably recruited in diverse roles, further implicating the importance of interoceptive processing in decision-making behavior. These findings reveal the neural signatures subserving alcohol costbenefit decision making, providing a foundation for future clinical applications of this paradigm and extending this approach to understanding the neural correlates of demand for other addictive commodities.

Neuropsychopharmacology (2014) 39, 1988-1995; doi:I0.1038/npp.2014.47; published online I6 April 2014

\section{INTRODUCTION}

Neuroeconomics integrates behavioral economics with cognitive neuroscience and has illuminated the neurobiological substrates underlying key aspects of decision making, including intertemporal choice (McClure et al, 2004), risk preferences (Tom et al, 2007), and gametheoretic interactions among individuals (Sanfey et al, 2003). Typically using functional magnetic resonance imaging (fMRI), neuroeconomics has provided qualitatively new perspectives on the nature of choice behavior, revealing the complex interactions between cognitive and affective brain systems in decisions involving a variety of different tradeoffs (Rangel et al, 2008). Moreover, a neuroeconomic approach has also been extended to clarify the differences in

*Correspondence: Dr J MacKillop, Department of Psychology, University of Georgia, 100 Hooper Street, Athens, GA 30605, USA, Tel: + I 706542 3033, Fax: + I 706542 3275, E-mail: jmackill@uga.edu Received 19 August 2013; revised 30 December 2013; accepted 21 February 2014; accepted article preview online 3 March 2014 brain activity that are associated with addictive disorders (Amlung et al, 2012a; Boettiger et al, 2007; Monterosso et al, 2007). Although incipient, this work leverages the extensive empirical research base applying behavioral economics to addictive disorders, all of which share a pathognomonic feature of overconsumption and are thus very well suited for examination using concepts from economics (for a review, see Murphy et al, 2012).

Despite the substantial progress to date, there has been relatively little study of the neural correlates of arguably the most fundamental form of economic tradeoff, the relationship between consumption of a commodity and its cost. In economics, this is referred to as demand and the relationship between consumption and cost-the elasticity of demand-is examined by plotting a demand curve of consumption across escalating prices (Hursh et al, 2005). Importantly, demand is typically not linear. Consumption is highest at minimal prices and is largely insensitive to initial price increases. As price rises, however, its effect becomes more powerful and, eventually, price entirely suppresses demand. Formally, the two periods of price sensitivity in the 
demand curve are referred to as inelastic demand, when consumption is relatively unaffected by price, and elastic demand, when consumption is substantially affected by price. These are followed by full price suppression.

In the context of understanding addictive disorders, demand curve analysis provides a model system for quantifying the value of a drug as a reinforcer. This is important because a core feature of alcohol and other substance use disorders is persistent overvaluation of drug reinforcement despite escalating negative consequences. This is also evident in empirical studies, where high alcohol demand has been consistently found to be significantly associated with severity of alcohol misuse (Gray and MacKillop, 2013; MacKillop et al, 2010a; Murphy and MacKillop, 2006; Murphy et al, 2009) and to predict treatment response (MacKillop and Murphy, 2007). Furthermore, indices of alcohol demand have been used to enhance the assessment of craving and clarify pharmacotherapy mechanisms (Bujarski et al, 2012; MacKillop et al, 2010b). Parallel findings have been found in behavioral studies on tobacco demand in relation to nicotine dependence (MacKillop et al, 2008, 2012; Murphy et al, 2011).

Little is known about the neural correlates of demandrelated decision making, both in general and in relation to addictive drugs. Two studies to date have used fMRI to investigate purchasing decisions for general commodities, finding initial evidence that the anterior insula and other subregions of the prefrontal cortex are recruited (Knutson and Bossaerts, 2007; Plassmann et al, 2007). However, both studies used diverse commodities and small numbers of prices, preventing a comprehensive examination of neural activity during demand-related decision making. No studies have systematically examined brain activity during decision making across the three periods of the canonical demand curve-maximum consumption during inelastic demand, reduced consumption during elastic demand, and the absence of consumption during suppressed demand.

This was the goal of the current study. We developed an fMRI alcohol demand paradigm to characterize the brain activity during cost-benefit decision making during each period of the demand curve. Participants made purchasing decisions for alcohol at varying levels of price in the MRI scanner and these choices determined how much alcohol they received in a subsequent bar laboratory session and how much money they kept for themselves. As an initial proof-of-concept investigation, the study sought to systematically characterize the patterns of neural activity across the alcohol demand curve and, in turn, to provide a foundation for future studies using fMRI to extend the findings in the existing behavioral literature.

\section{MATERIALS AND METHODS}

\section{Eligibility Criteria and Participants}

Inclusion criteria were: (1) 21-31 years of age; (2) heavy drinker status (ie, $21+$ standard drinks/week); (3) righthanded; (4) computer literacy (ie, at least weekly use of a personal computer/smart phone); and (5) male sex (given power limitations, only one sex was selected to eliminate possible sex differences; males were selected based on higher prevalence rates of AUDs; Khan et al, 2013). Heavy drinkers were selected because the behavioral boundary conditions for alcohol demand decision making are well established (ie, robust demand for alcohol at very low prices, attenuated demand at intermediate prices, and full suppression at high prices; Amlung et al, 2012b). Furthermore, heavy drinkers were considered an optimal sample for validation of the paradigm as their performance would be relevant to higher and lower drinking samples in future studies. Weekly alcohol consumption was assessed using a 28-day Timeline Follow-Back interview (Sobell et al, 1979). Exclusion criteria were: (1) seeking treatment for alcohol problems in the past 90 days; (2) currently taking psychotropic medications; (3) current DSM-IV substance use disorder (SUD), other than an alcohol use disorder or nicotine dependence, or other Axis I disorders; (4) any contraindications for MRI scanning; (5) history of serious head injury; and (6) attending session with a positive breath alcohol level (Intoximeters Alco-sensor IV, St Louis, MO).

In all, 26 participants were enrolled, but 2 were subsequently excluded because of noncompliance or absence of an event category (see below). Participants were primarily young adults $(\mathrm{M}=22.58, \mathrm{SD}=2.62)$ of European ancestry (79\%, 8\% Asian, 13\% mixed race; 13\% Hispanic ethnicity) and had a median income of $\$ 45-60000$ annually. The sample was characterized by heavy drinking (drinks/week $\mathrm{M}=34.26, \mathrm{SD}=11.30$ ), but relatively low rates of other substance use. Five were smokers (mean cigarettes/ day $=6.2$ ), four reported weekly marijuana use, and one reported weekly cocaine use.

\section{Experimental Protocols and fMRI Paradigm}

Participants were initially screened via a telephone interview and eligible participants were then invited for an in-person screen. There, participants were given an overview of the study and those interested were enrolled upon providing written informed consent. At that point, participants were given an orientation to the 8 -h protocol that comprised the MRI scan and the alcohol selfadministration/recovery periods. To ensure participants were fully informed, they were also introduced to the bar laboratory, drink sizes, and the recovery room during the in-person screen.

At the start of the fMRI-Bar Lab protocol, research assistants confirmed sobriety and MRI compatibility, and reviewed the study procedures with the participants. Participants then underwent a 60-min MRI scan, including five runs of an Alcohol Purchase Task (APT) fMRI paradigm. The event-related paradigm consisted of choices of how many drinks they would like to 'buy' at varying levels of price. In each run, 22 randomized prices were assessed (duration $=7.2 \mathrm{~min}$ ), ranging from $\$ 0.01$ to $\$ 15.00$ / drink (Supplementary Materials and Supplementary Table S1). For all choices, participants had a $\$ 15$ 'bar tab' to be allocated to drink purchases or not spent and kept by the participant. The drinks available were the participants' typical alcoholic beverages and, based on previous bar lab self-administration paradigms (see, eg, Drobes et al, 2003), each person could purchase a maximum of 8 'mini-drinks,' each approximately half the size of standard drinks. The total amount of alcohol available was a volume sufficient to raise their blood alcohol level to $0.10 \%$ based on age, sex, 
weight, and height, calculated via standard calculations (Brick, 2006). Smokers were permitted to smoke 1 hour before the scan to avoid withdrawal.

For maximum clarity, the fMRI paradigm divided the decision-making process into two epochs, a 'Decide' phase (fixed duration $=4 \mathrm{~s}$ ) in which participants were instructed to mentally choose how much they would like to drink, and an 'Input' phase (lasting $\leqslant 6 \mathrm{~s}$ ) in which they actually toggled between options to indicate their choice. During Decide, the prices and options were shown but the response box did not register any input. During Input, a center starting point was outlined in green and participants moved the green outline left or right to the preferred option using their right hand. The selection was then confirmed by pressing a button with their left hand. This two-phase strategy permitted equal durations during deliberation for each choice and dissociation of deliberation from subsequent motor selection. Following selection, the display changed to the jittered interstimulus interval (ISI) screen ( $\mathrm{M}$ duration $=8 \mathrm{~s}$; range $=1-15 \mathrm{~s})$, an active baseline condition with numbers replaced with Xs to match the stimulus properties of the active trials.

To yoke the choices to actual outcomes, participants received one randomly selected outcome from their choices at the conclusion of the scan. They were then transported by university vehicle to the bar laboratory, $\sim 1$ mile away. The 60 -min self-administration period commenced and participants received their randomly selected outcome (ie, up to 8 mini-drinks and any remaining change from their bar tab). Participants also had access to a bottle of spring water to control for general thirst. During this period, participants were allowed to drink as much alcohol as they received from the task ad libitum. At the end of the self-administration period, all participants were required to remain in the laboratory for a $4-\mathrm{h}$ recovery period, regardless of whether alcohol was received or consumed. The recovery environment was a neutral laboratory room containing a couch, desk, and chair. Participants were permitted to read and use the Internet/telephones during the recovery period. The maximum allowable BrAC for departure at the end of the session was $0.04 \%(\mathrm{M}=0.013, \mathrm{SD}=0.014)$. Participants were compensated $\$ 15 / \mathrm{h}$, paid by check $\sim 3$ weeks following completion. All procedures were IRB approved and conformed to NIAAA ethical recommendations for alcohol administration.

\section{Primary Analytic Strategy}

Events (choices) were trichotomized into one of the three mutually exclusive categories according to the three periods of the prototypic demand curve: 'Inelastic,' 'Elastic,' and 'Suppressed.' Inelastic choices were defined as choices for a participant's personal maximum consumption, typically at $1 \varnothing$ and other very low prices; these choices putatively reflected consumption that was independent of the associated costs. Elastic choices were defined as choices in which consumption was less than maximum but remained greater than zero, as these choices putatively reflected consumption that was affected by response cost (price), but not eliminated. Suppressed choices were defined as choices for no alcohol, as these choices putatively reflected complete price control over consumption. These categories were intended to capture the three possible combinations of cost-benefit decision making. This is depicted in Figure 1a: Inelastic choices reflected perceived benefits of drinking outweighing the associated costs; Elastic choices reflected perceived benefits and costs of drinking actively competing; and Suppressed choices reflected the perception of costs outweighing drinking.

Imaging data were collected at the University of Georgia Bio-Imaging Research Center using a GE Signa HDx 3-Tesla scanner. Imaging parameters and initial data processing are provided in Supplementary Materials. Functional imaging data analyses were conducted in Analysis of Functional NeuroImages (AFNI) software (Cox, 1996). Group analysis was executed using general linear model (GLM) $\beta$ coefficients. Differential activation by choice type was examined using an empirically defined disjunction mask approach (Ballard and Knutson, 2009; MacKillop et al, 2012). Specifically, a region of interest (ROI) mask was generated that comprised all regions that were significantly activated or deactivated in any of the three choice conditions (Inelastic, Elastic, and Suppressed).

To correct for multiple comparisons, we first estimated the spatial smoothness in the data using the residuals from the multiple regression and then inputted the average smoothness in the X, Y, and Z planes to AlphaSim (Ward, 2000) to obtain the minimum cluster extent needed for a family-wise $\alpha<0.05$. This yielded a minimum cluster size of 9 voxels $(>385 \mu \mathrm{m})$ and an uncorrected significance threshold of $p<0.0001$. For initial masks, small numbers of ROIs were very large and subsumed multiple discrete regions, and hence $p$-values were successively increased to identify functionally distinct regions. This was determined before any further analysis by reviewing AFNI anatomical proportionate designations for subsumed ROIs (for details, see Supplementary Materials). Identification of regions that exhibited differential neural activity by choice type was then conducted using average blood oxygen level-dependent (BOLD) signal (GLM $\beta$ coefficients) per ROI per individual as the dependent variable. Specifically, omnibus one-way three-level (Inelastic, Elastic, and Suppressed) withinsubject analyses of variance were conducted, with significant effects followed-up with pairwise $t$-tests to identify specific patterns. Further multiple comparison correction was implemented by only following-up ROIs that had omnibus effects that survived a false discovery rate correction, $q=0.05$ (Benjamini and Hochberg, 1995). For descriptive purposes, voxelwise one-sample $t$-tests $v s 0$ were conducted to identify regions of activation/deactivation per condition. These complete activation maps, the omnibus ROI activity effects, and follow-up contrasts are reported in Supplementary Materials.

\section{RESULTS}

\section{Behavioral Results}

Behavioral performance was prototypic, with the aggregated demand curve exhibiting inelastic, elastic, and suppressed periods (Figure 1b). Following trichotomization, substantial differences were evident between the three choice category preferences (Figure 1c) that were all significantly different from each other $\left(\mathrm{F}(2,46)=350.97, p<0.001, \eta_{\mathrm{p}}^{2}=0.94\right.$, 


\begin{tabular}{|l|c|c|}
\hline \multicolumn{1}{|c}{ Choice Type } & \multicolumn{1}{c}{$\begin{array}{c}\text { Perception of } \\
\text { Benefit }\end{array}$} & $\begin{array}{c}\text { Perception } \\
\text { of Cost }\end{array}$ \\
\hline Inelastic Demand (Maximum Consumption) & + & - \\
\hline Elastic Demand $(0<$ Consumption < Max) & + & + \\
\hline Suppressed Demand (Consumption $=0$ ) & - & + \\
\hline
\end{tabular}

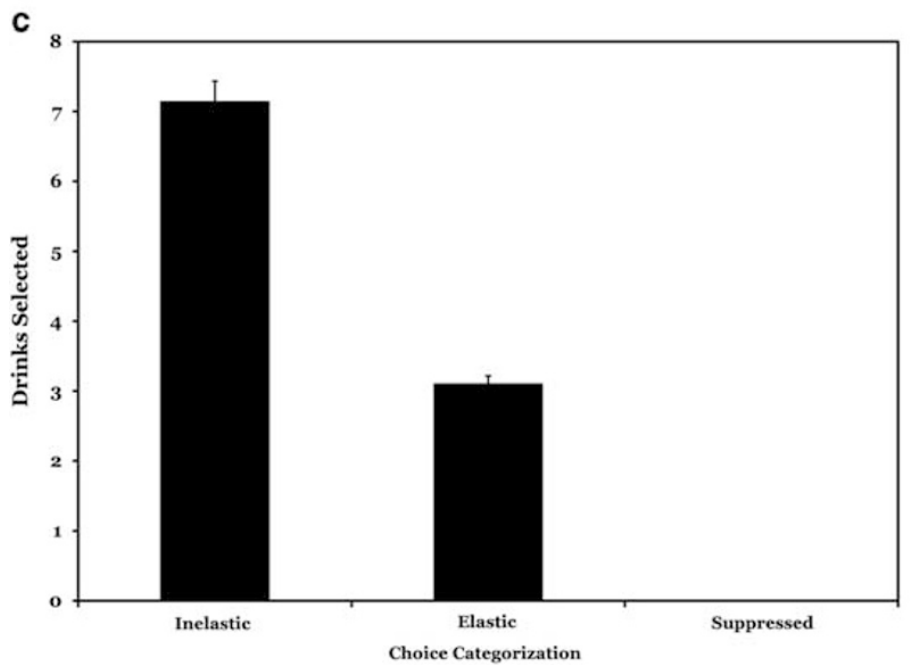

b

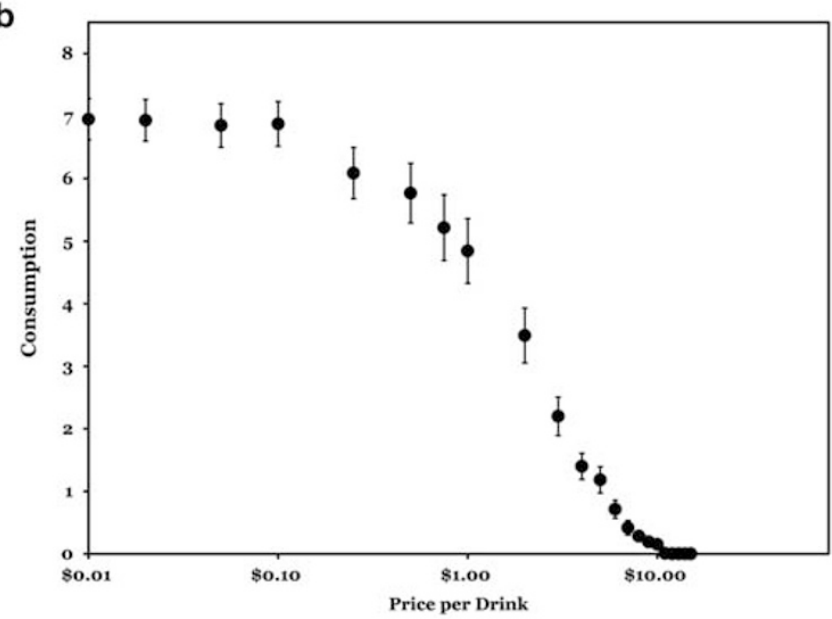

d

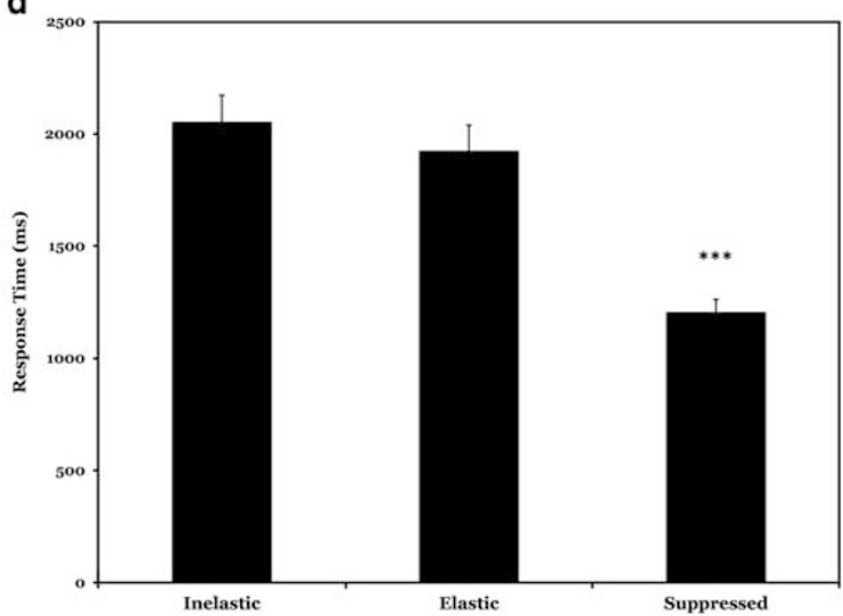

Figure I Behavioral performance during an $\mathrm{PMRI}$ alcohol purchase task. (a) The cost-benefit designations associated with the trichotomous event designation. (b) The aggregated empirical alcohol demand curve based on participant choices. (c) The average number of drinks associated with the trichotomous categorization; all choice types were significantly different from each other $(p ' s<0.001)$. Error bars reflect SEM. (d) Reaction time during the three choice categories; significant differences are present between Inelastic and Elastic choices compared with Suppressed choices but not relative to each other $(* * * * * 0.001)$.

contrast $p s<0.001$ ). In terms of reaction time (Figure 1d), a significant main effect of choice type on response time was evident $\left(\mathrm{F}(2,46)=46.29, p<0.001, \eta_{\mathrm{p}}^{2}=0.67\right.$, $)$; participants responded faster during Suppressed choices compared with Inelastic and Elastic choices ( $p$ 's $<0.001)$, but the latter two did not significantly differ $(p=0.26)$.

\section{Neural Correlates of Alcohol Demand during the Decide Epoch}

During the Decide epoch, 24 clusters of significant activity were present across choice types. Eight of these did not significantly discriminate between choice type, reflecting cognitive processing that was not specific to a choice type (coordinates are provided in Supplementary Table S2). Of the discriminating ROIs, two common profiles of activation were present and several regions exhibited unique patterns of activity. The first common profile comprised six ROIs that reflected significantly greater neural activity during
Inelastic and Elastic choices compared with Suppressed choices (Figure 2a). This was termed the 'DRINK' profile because it reflected both categories of choices that resulted in any alcohol being distinct from decisions that resulted in none. The regions exhibiting the DRINK profile included bilateral posterior parietal cortex (PPC), right medial prefrontal cortex (mePFC) and dorsolateral PFC (dlPFC), and left posterior cingulate cortex (PCC) and left anterior insula.

The second common pattern of activity was of regions exhibiting significantly different neural activity during Elastic choices compared with both Inelastic and Suppressed choices (Figure $2 \mathrm{~b}$ ). This profile was termed the 'DRINK?' profile as it reflected selectively greater activity when individuals were choosing to drink, but at lower levels than their maximum level (ie, drinking preferences partially suppressed by cost). The DRINK? ROIs comprised significantly greater activation in the right anterior insula, and significantly greater deactivation in multiple regions, 

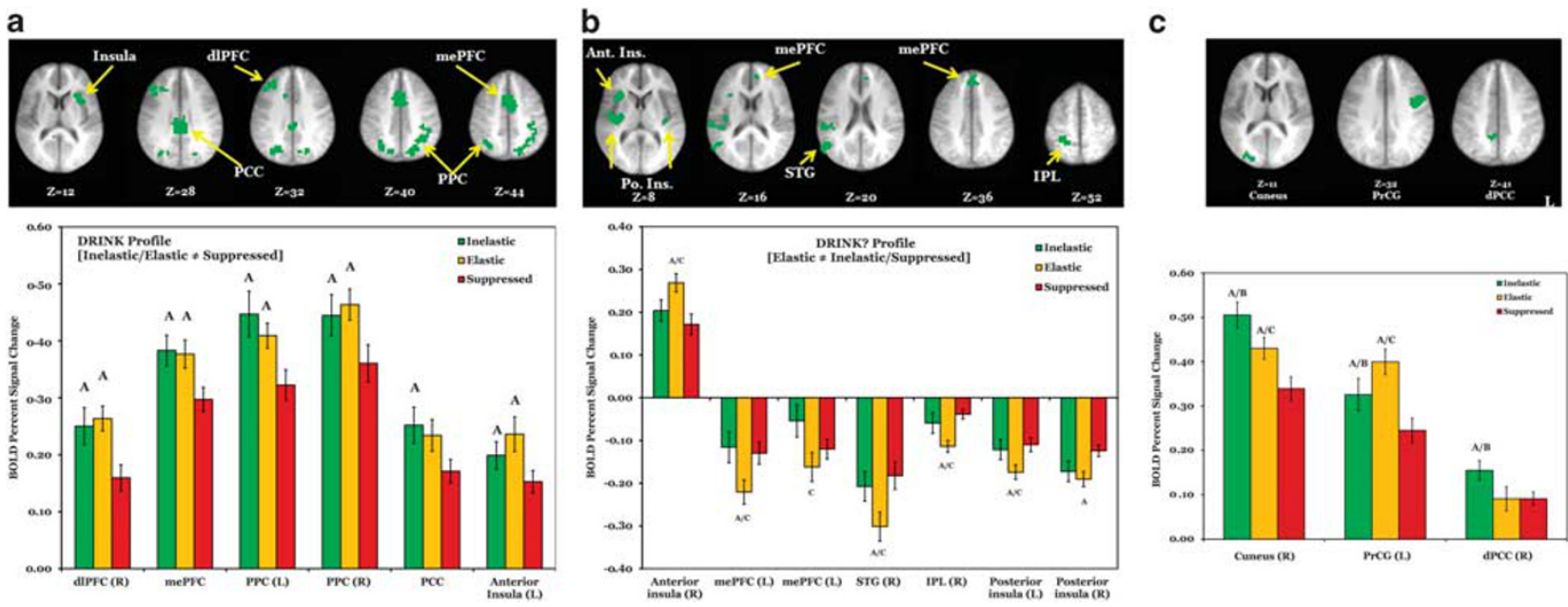

Figure 2 BOLD signal associated with differential activity during the Decide epoch of alcohol demand decision-making. (a) The regions (top) and BOLD differences (bottom) associated with the 'DRINK' profile (ie, greater activity in the two categories resulting in any alcohol vs choices for none). (b) The regions and BOLD differences associated with the 'DRINK?' profile (ie, selectively different activity during the Elastic choices). (c) Regions and BOLD differences associated with unique profiles of activity, including general price sensitivity and maximum consumption preference. Brain maps present the anatomical disjunction mask regions of interest. Statistical significance of BOLD signal magnitude differences is denoted as follows: A, significantly different from Suppressed; B, significantly different from Elastic; C, significantly different from Inelastic.

including the mePFC, superior temporal gyrus (STG), inferior parietal lobule (IPL), and posterior insula.

Three ROIs exhibited unique patterns of activity (Figure 2c). Two exhibited significant differences across all three choice types, but in different configurations. Activation in the cuneus scaled to the alcohol demand curve, with the highest activation during Inelastic choices, significantly lower activation during Elastic choices, and significantly lower activation still during Suppressed choices. In contrast, precentral gyrus (PrCG) differentiated among the choice types, but exhibited the greatest activity during Elastic choices, followed by Inelastic choices, and then Suppressed choices. Compared with both Elastic and Suppressed choices, PCC activity was significantly higher during Inelastic choices, suggesting it was uniquely associated with motivation for maximum alcohol.

\section{Neural Correlates of Alcohol Demand during the Input Epoch}

During the Input epoch, 21 clusters of significant activity were observed across choice types, but 6 were nondiscriminating across choice types (Supplementary Table S2). Among the discriminating ROIs, the majority exhibited the previously observed DRINK? profile, with BOLD signal being significantly greater during Elastic choices compared with both Inelastic and Suppressed choices (Figure 3a). This was most evident bilaterally in dlPFC, PrCG, angular gyrus, and striatum, in right fusiform gyrus, and in PCC along the midline. The cerebellar uvula and medial occipital gyrus exhibited qualitatively similar activation. The three remaining ROIs were variants of the DRINK profile (Figure $3 \mathrm{~b}$ ), but with important differences. Significantly greater activation was present in the anterior cingulate cortex (ACC) and right cuneus during Inelastic and Elastic choices as compared with Suppressed, but activity in the right anterior insula exhibited an inverse profile, with significantly greater activation during Suppressed choices as compared with the other two choices.

\section{DISCUSSION}

The current study sought to characterize the neural correlates of alcohol cost-benefit decision making (ie, alcohol demand). Using the canonical demand curve as a framework for classifying choices as unaffected by price (Inelastic), affected by price (Elastic), and entirely abolished by price (Suppressed), we identified common and unique patterns of brain activity associated with the different costbenefit choice profiles. During the deliberation phase (Decide), we observed significant differential activation that predominantly conformed to two patterns. The DRINK pattern comprised significantly greater neural activity during Inelastic and Elastic choices compared with Suppressed choices (ie, choices for any alcohol being different from those that were for none). During these choices, the regions exhibiting differential activity included areas associated with attention and intentionality (bilateral PPC; Desmurget et al, 2009; Shomstein, 2012); deliberation and decisional balance (mePFC and dlPFC; Rushworth et al, 2011); introspection and balancing internally and externally directed cognition (PCC; Leech et al, 2012; Vogt and Laureys, 2005); and drug cravings (anterior insula; Naqvi and Bechara, 2009).

The second profile, DRINK?, comprised regions exhibiting significantly different neural activity during Elastic choices compared with both Inelastic and Suppressed choices. Here, differentially greater activation was evident in right anterior insula, whereas differentially greater deactivations were evident in several regions consistent with disengagement of the default mode network and 
a
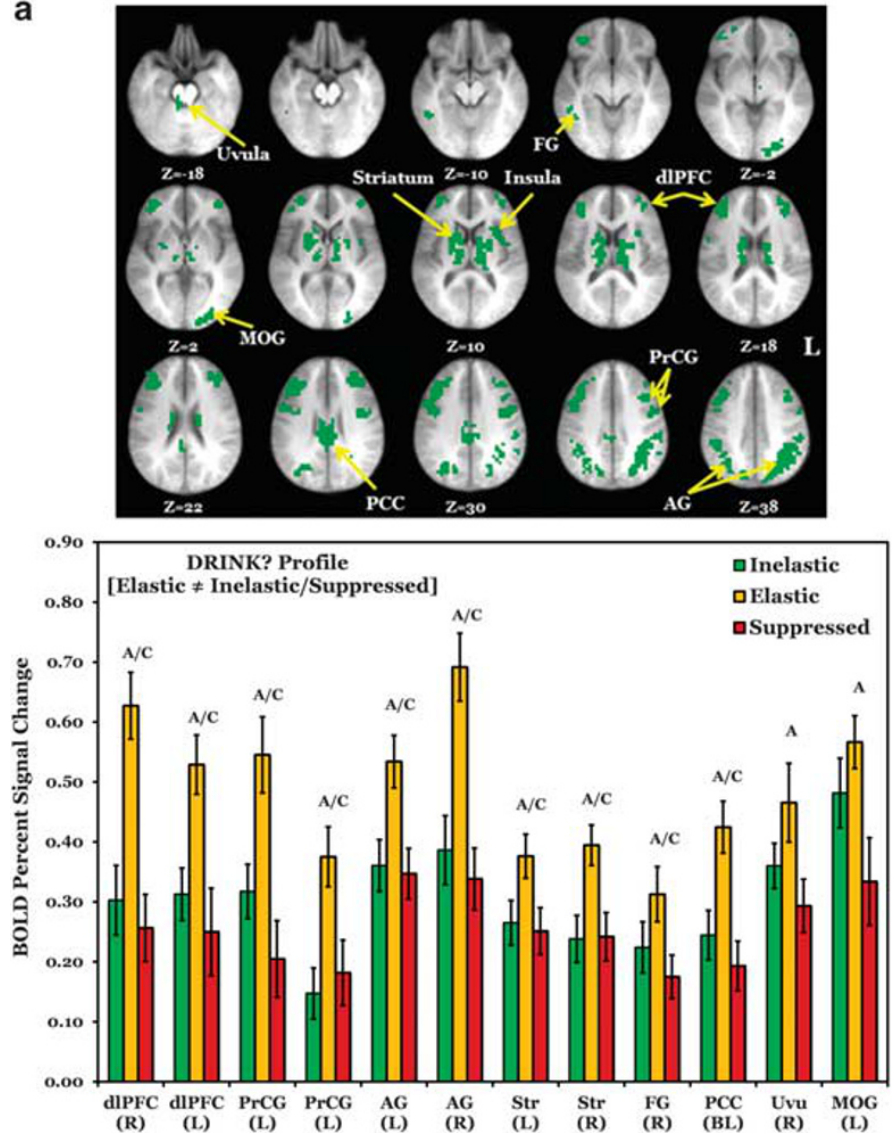
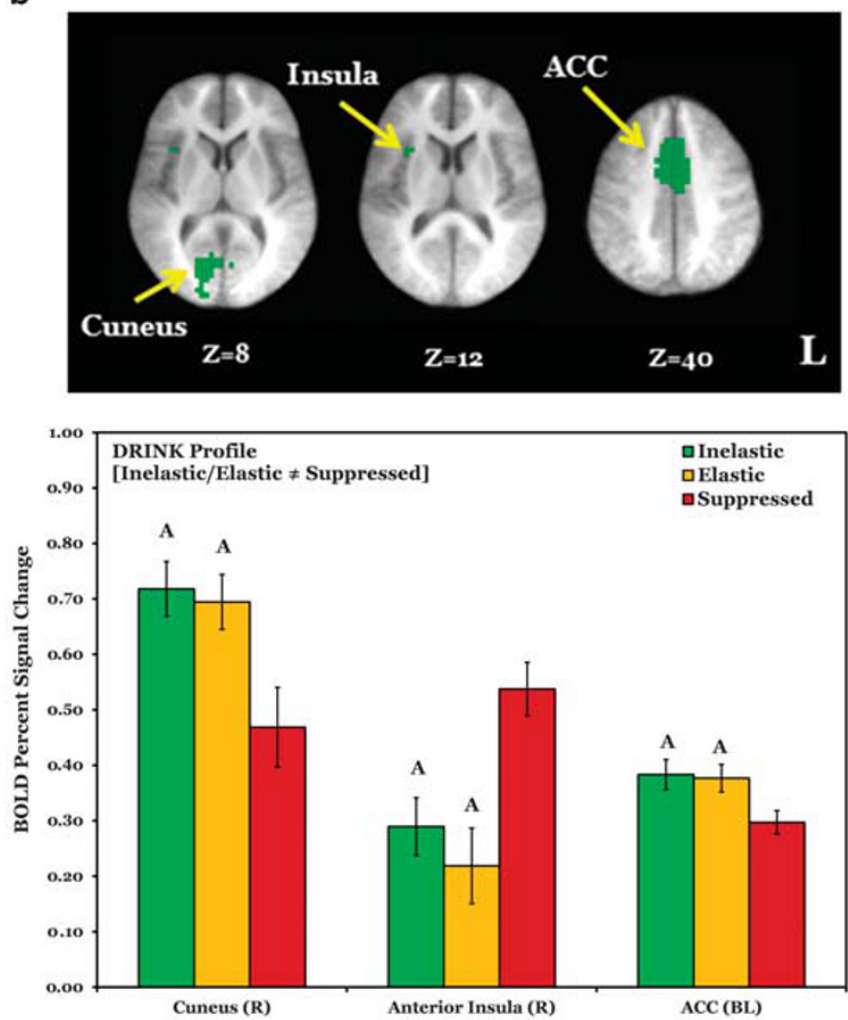

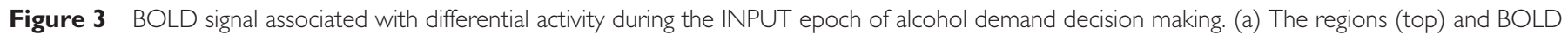
differences (bottom) associated with the 'DRINK?' profile (ie, selectively different activity during the Elastic choices). (b) Regions and BOLD differences

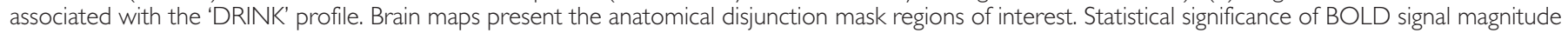
differences is denoted as follows: A, significantly different from Suppressed; C, significantly different from Inelastic.

allocation of directed attention (Tomasi et al, 2006; Whitfield-Gabrieli and Ford, 2012).

Two additional ROIs were of considerable interest during the Decide phase. Activation in the cuneus was significantly different across the three choice types, mapping onto the topography of the alcohol demand curve. This suggests that the cuneus plays a crucial role in price sensitivity attributions and is consistent with previous studies implicating it with both the salience and incentive value of a commodity (Engelmann et al, 2009; Litt et al, 2011). Also of interest, dorsal PCC was significantly higher during Inelastic choices compared with both Elastic and Suppressed choices, and was the only region associated with preferences for maximum consumption. This is consistent with its implication in attributing value to a commodity (Litt et al, 2011) and suggests the dorsal PCC may be particularly sensitive to contingencies interpreted as bargains.

During the Input phase, when participants executed their choices, the same two patterns of differential activation predominantly emerged. The most common pattern was the DRINK? profile, in which selectively greater activity was present during Elastic choices compared with both Inelastic and Suppressed choices. Differentially greater activation was present in regions associated with cognitive deliberation and integration (dlPFC and PCC; Lee and Seo, 2007;
Leech et al, 2012), mathematical calculations (angular gyrus; Zamarian et al, 2009), and motivational drive/ cravings (striatum and insula; Naqvi and Bechara, 2009; Shohamy, 2011). Thus, during Elastic choices, participants exhibited significantly greater brain activity in diverse frontostriatal regions reflecting processing of the total cost of alcohol, the subjective value of alcohol, and the tension between these two domains. This supports the notion that cost-benefit decisions reflect underlying processes that are both objective (eg, mental arithmetic to calculate the cost of a number of drinks) and subjective (eg, the individual's perceived value of alcohol).

Taken together, these findings reveal the diverse neuroanatomical regions that are recruited in processing fundamental cost-benefit decision making. What emerges suggests critical roles for frontoparietal and frontostriatal regions that are relatively well established as subserving aspects of reward processing and decision making, and also a number of other brain regions that are increasingly implicated in decision making. For example, the selective activation in dorsal PCC provides further evidence of its integrative role in decision making that balances internal and external contingencies (Leech et al, 2012), and the scaling of brain activation in the cuneus to alcohol across the three periods of the demand curve further confirms its role in encoding value (Litt et al, 2011). 
These data also provide further evidence to suggest that the anterior insula plays a critical role in cost-benefit decision making. During the Decide epoch, the anterior insula was differentially active during DRINK and DRINK? choices and, during the Input epoch, the anterior insula was selectively more active during choices of not to drink. These findings are compatible with the hypothesis that the anterior insula plays an important role in the distributed network subserving drug motivation (Naqvi and Bechara, 2009). In addition, these findings are consistent with evidence that the insula is recruited when commodities are perceived to be overpriced or transactions are perceived to be unfair (King-Casas et al, 2008; Knutson and Greer, 2008; Sanfey et al, 2003). In the context of the current study, the anterior insula appeared to register how much the participants 'felt' drinking was worth at a given price.

Importantly, the current findings should be considered in the context of the study's strengths and limitations. In terms of strengths, the study was the first to use fMRI to characterize drug demand and used an established behavioral paradigm including actual units of money and the participants' preferred alcohol. Furthermore, the behavioral performance met all the necessary boundary conditions for examining neural activity across the demand curve (ie, high initial demand, reduction in response to increases in price, and ultimately complete suppression of consumption). On the other hand, as an initial validation study of the neural correlates of alcohol demand, the study had a relatively modest sample size and, by virtue of its within-subject design, uniformly heavy drinkers. As such, we cannot address differences between the current sample and either lower-risk or higher-risk groups. Similarly, a limitation of the study is that the participants were all male, making the generalizability of these findings to females unclear at this point. Although we would predict parallel activation patterns, sex differences may be present and this is fundamentally an empirical question. Future studies will be necessary to both confirm the activation patterns reported here, to identify differences in neural activity between groups varying in AUD severity, and to investigate brain activity in both males and females systematically.

More broadly, another consideration is that the study was based on the general assumption of quantitative differences in demand-related brain activity across commodities, not qualitative differences (ie, differences in scale but not in kind). This is a common neuroeconomic assumption and has been supported empirically in evidence of largely overlapping brain regions in intertemporal tradeoffs for monetary rewards and food rewards (McClure et al, 2007). However, another future direction will be verifying this assumption and evaluating whether qualitatively different patterns of brain activity are observable across addictive and control commodities. Finally, it is worth noting that the neural activity observed was inherently contextualized within the amount of the bar tab that was almost certainly less than would be available to the participants in real-world drinking. These parameters appear to have worked well, as the resulting demand curve conformed to the prototypic shape and intended numbers of events; nonetheless, it is worth investigating alternative parameters in future studies.

In sum, the current study identified the common and unique patterns of brain activity associated with distinct alcohol cost-benefit choice profiles. Characterizing the neural correlates of alcohol demand provides a foundation for diverse applications using this paradigm, including between-group comparisons (eg, Murphy and MacKillop, 2006), enhanced measurement of craving (eg, MacKillop et al, 2010b), and clarification of pharmacotherapy mechanisms (eg, Bujarski et al, 2012). More broadly, the current findings also provide an empirical basis for examining the neural profiles that subserve demand decision making in other disorders of overconsumption, including other forms of drug addiction, pathological gambling, and obesity.

\section{FUNDING AND DISCLOSURE}

The authors declare no conflict of interest.

\section{ACKNOWLEDGEMENTS}

We are very grateful for the contributions of the Research Assistants of the UGA Experimental and Clinical Psychopharmacology Laboratory and to Max Owens for editorial assistance. We gratefully acknowledge financial support from NIH grants K23 AA016936 and P30 DA027827; a University of Georgia Research Foundation Faculty grant; and paradigm development hours from the UGA BioImaging Research Center. The funders had no role in study design, data collection, or manuscript preparation.

\section{REFERENCES}

Amlung M, Sweet LH, Acker J, Brown CL, Mackillop J (2012a). Dissociable brain signatures of choice conflict and immediate reward preferences in alcohol use disorders. Addict Biol doi:10.1111/adb.12017 (http://www.ncbi.nlm.nih.gov/pubmed/ 23231650).

Amlung MT, Acker J, Stojek MK, Murphy JG, MacKillop J (2012b). Is talk 'cheap'? An initial investigation of the equivalence of alcohol purchase task performance for hypothetical and actual rewards. Alcohol Clin Exp Res 36: 716-724.

Ballard K, Knutson B (2009). Dissociable neural representations of future reward magnitude and delay during temporal discounting. Neuroimage 45: 143-150.

Benjamini Y, Hochberg Y (1995). Controlling the false discovery rate: a practical and powerful approach to multiple testing. $J$ R Stat Soc Ser B 57: 289-300.

Boettiger CA, Mitchell JM, Tavares VC, Robertson M, Joslyn G, D'Esposito $M$ et al (2007). Immediate reward bias in humans: fronto-parietal networks and a role for the catechol-O-methyltransferase 158(Val/Val) genotype. J Neurosci 27: 14383-14391.

Brick J (2006). Standardization of alcohol calculations in research. Alcohol Clin Exp Res 30: 1276-1287.

Bujarski S, MacKillop J, Ray LA (2012). Understanding naltrexone mechanism of action and pharmacogenetics in Asian Americans via behavioral economics: a preliminary study. Exp Clin Psychopharmacol 20: 181-190.

Cox RW (1996). AFNI: software for analysis and visualization of functional magnetic resonance neuroimages. Comput Biomed Res 29: 162-173.

Desmurget M, Reilly KT, Richard N, Szathmari A, Mottolese C, Sirigu A (2009). Movement intention after parietal cortex stimulation in humans. Science 324: 811-813.

Drobes DJ, Anton RF, Thomas SE, Voronin K (2003). A clinical laboratory paradigm for evaluating medication effects on alcohol 
consumption: naltrexone and nalmefene. Neuropsychopharmacology 28: 755-764.

Engelmann JB, Capra CM, Noussair C, Berns GS (2009). Expert financial advice neurobiologically 'Offloads' financial decisionmaking under risk. PLoS One 4: e4957.

Gray J, MacKillop J (2013). Interrelationships among individual differences in alcohol demand, impulsivity, and alcohol misuse. Psychol Addict Behav (http://www.ncbi.nlm.nih.gov/ pubmed/23915370).

Hursh SR, Galuska CM, Winger G, Woods JH (2005). The economics of drug abuse: a quantitative assessment of drug demand. Mol Interv 5: 20-28.

Khan S, Okuda M, Hasin DS, Secades-Villa R, Keyes K, Lin K-H et al (2013). Gender differences in lifetime alcohol dependence: results from the national epidemiologic survey on alcohol and related conditions. Alcohol Clin Exp Res 37: 1696-1705.

King-Casas B, Sharp C, Lomax-Bream L, Lohrenz T, Fonagy P, Montague PR (2008). The rupture and repair of cooperation in borderline personality disorder. Science 321: 806-810.

Knutson B, Bossaerts P (2007). Neural antecedents of financial decisions. J Neurosci 27: 8174-8177.

Knutson B, Greer SM (2008). Anticipatory affect: neural correlates and consequences for choice. Philos Trans R Soc Lond B Biol Sci 363: 3771-3786.

Lee D, Seo H (2007). Mechanisms of reinforcement learning and decision making in the primate dorsolateral prefrontal cortex. Ann N Y Acad Sci 1104: 108-122.

Leech R, Braga R, Sharp DJ (2012). Echoes of the brain within the posterior cingulate cortex. J Neurosci 32: 215-222.

Litt A, Plassmann H, Shiv B, Rangel A (2011). Dissociating valuation and saliency signals during decision-making. Cereb Cortex 21: 95-102.

MacKillop J, Brown CL, Stojek MK, Murphy CM, Sweet L, Niaura RS (2012). Behavioral economic analysis of withdrawal- and cueelicited craving for tobacco: an initial investigation. Nicotine Tob Res 14: 1426-1434.

MacKillop J, Miranda RJ, Monti PM, Ray LA, Murphy JG, Tidey JW et al (2010a). Alcohol demand, delayed reward discounting, and craving in relation to drinking and alcohol use disorders. J Abnorm Psychol 119: 115-125.

MacKillop J, Murphy JG (2007). A behavioral economic measure of demand for alcohol predicts brief intervention outcomes. Drug Alcohol Depend 89: 227-233.

MacKillop J, Murphy JG, Ray LA, Eisenberg DT, Lisman SA, Lum JK et al (2008). Further validation of a cigarette purchase task for assessing the relative reinforcing efficacy of nicotine in college smokers. Exp Clin Psychopharmacol 16: 57-65.

MacKillop J, O'Hagen S, Lisman SA, Murphy JG, Ray LA, Tidey JW et al (2010b). Behavioral economic analysis of cue-elicited craving for alcohol. Addiction 105: 1599-1607.

McClure SM, Ericson KM, Laibson G, Cohen JD (2007). Time discounting for primary rewards. J Neurosci 27: 5796-804.

McClure SM, Laibson DI, Loewenstein G, Cohen JD (2004). Separate neural systems value immediate and delayed monetary rewards. Science $(80-$ ) 306: 503-507.

Monterosso JR, Ainslie G, Xu J, Cordova X, Domier CP, London ED (2007). Frontoparietal cortical activity of methamphetamine- dependent and comparison subjects performing a delay discounting task. Hum Brain Mapp 28: 383-393.

Murphy J, MacKillop J (2006). Relative reinforcing efficacy of alcohol among college student drinkers. Exp Clin Psychopharmacol 14: 219-227.

Murphy JG, MacKillop J, Skidmore JR, Pederson AA (2009). Reliability and validity of a demand curve measure of alcohol reinforcement. Exp Clin Psychopharmacol 17: 396-404.

Murphy JG, Mackillop J, Tidey JW, Brazil LA, Colby SM (2011). Validity of a demand curve measure of nicotine reinforcement with adolescent smokers. Drug Alcohol Depend 113: 207-214.

Murphy JG, MacKillop J, Vuchinich RE, Tucker JA (2012). Behavioral economic concepts in the analysis of substance abuse. In S. Walters, J. Morgenstern, and F. Rotgers (eds.) Treating Substance Abuse, Theory and Technique 3rd edn. Guilford Press: New York, pp 48-80.

Naqvi NH, Bechara A (2009). The hidden island of addiction: the insula. Trends Neurosci 32: 56-67.

Plassmann H, O’Doherty J, Rangel A (2007). Orbitofrontal cortex encodes willingness to pay in everyday economic transactions. J Neurosci 27: 9984-9988.

Rangel A, Camerer C, Montague PR (2008). A framework for studying the neurobiology of value-based decision making. Nat Rev Neurosci 9: 545-556.

Rushworth MFS, Noonan MP, Boorman ED, Walton ME, Behrens TE (2011). Frontal cortex and reward-guided learning and decision-making. Neuron 70: 1054-1069.

Sanfey AG, Rilling JK, Aronson JA, Nystrom LE, Cohen JD (2003). The neural basis of economic decision-making in the Ultimatum Game. Science 300: 1755-1758.

Shohamy D (2011). Learning and motivation in the human striatum. Curr Opin Neurobiol 21: 408-414.

Shomstein S (2012). Cognitive functions of the posterior parietal cortex: top-down and bottom-up attentional control. Front Integr Neurosci 6: 38.

Sobell LC, Maisto SA, Sobell MB, Cooper AM (1979). Reliability of alcohol abusers' self-reports of drinking behavior. Behav Res Ther 17: 157-160.

Tom SM, Fox CR, Trepel C, Poldrack RA (2007). The neural basis of loss aversion in decision-making under risk. Science 315: 515-518.

Tomasi D, Ernst T, Caparelli EC, Chang L (2006). Common deactivation patterns during working memory and visual attention tasks: an intra-subject fMRI study at 4 Tesla. Hum Brain Mapp 27: 694-705.

Vogt BA, Laureys S (2005). Posterior cingulate, precuneal and retrosplenial cortices: cytology and components of the neural network correlates of consciousness. Prog Brain Res 150: 205-217.

Ward D (2000): Simultaneous Inference for fMRI Data. 16 (http:// afni.nimh.nih.gov/pub/dist/doc/manual/AlphaSim.pdf).

Whitfield-Gabrieli S, Ford JM (2012). Default mode network activity and connectivity in psychopathology. Annu Rev Clin Psychol 8: 49-76.

Zamarian L, Ischebeck A, Delazer M (2009). Neuroscience of learning arithmetic-evidence from brain imaging studies. Neurosci Biobehav Rev 33: 909-925.

Supplementary Information accompanies the paper on the Neuropsychopharmacology website (http://www.nature.com/npp) 